\title{
Immediate and long-term consequences of COVID-19 infections for the development of neurological disease
}

\author{
Michael T. Heneka ${ }^{1,2,3^{*}}$ D, Douglas Golenbock², Eicke Latz ${ }^{2,3,4}$, Dave Morgan ${ }^{5}$ and Robert Brown ${ }^{6}$
}

\begin{abstract}
Increasing evidence suggests that infection with Sars-CoV-2 causes neurological deficits in a substantial proportion of affected patients. While these symptoms arise acutely during the course of infection, less is known about the possible longterm consequences for the brain. Severely affected COVID-19 cases experience high levels of proinflammatory cytokines and acute respiratory dysfunction and often require assisted ventilation. All these factors have been suggested to cause cognitive decline. Pathogenetically, this may result from direct negative effects of the immune reaction, acceleration or aggravation of pre-existing cognitive deficits, or de novo induction of a neurodegenerative disease. This article summarizes the current understanding of neurological symptoms of COVID-19 and hypothesizes that affected patients may be at higher risk of developing cognitive decline after overcoming the primary COVID-19 infection. A structured prospective evaluation should analyze the likelihood, time course, and severity of cognitive impairment following the COVID-19 pandemic.
\end{abstract}

Keywords: Systemic inflammation, NLRP3 inflammasome, Cytokine, Cognition, Neurodegeneration, Neuroinflammation, Decline

\section{Background}

The COVID-19 pandemic represents an unprecedented immediate but also persisting threat to our health care systems. The immediate urgency is clear, as the total number of affected patients exceeds most of the health care system capacities worldwide, particularly for the treatment of patients requiring assisted ventilation. Less immediately apparent, but potentially very significant are long-term consequences of COVID-19 infections.

During the acute phase of COVID-19 infection, about $36 \%$ of cases develop neurological symptoms of which $25 \%$ can be attributed to the direct involvement of the central nervous system. The main symptoms include but are not restricted to dizziness, headache, impaired

\footnotetext{
* Correspondence: michael.heneka@ukbonn.de

'Department of Neurodegenerative Disease and Geriatric Psychiatry,

University of Bonn, Bonn, Germany

${ }^{2}$ German Center for Neurodegenerative Disease, Bonn, Germany

Full list of author information is available at the end of the article
}

consciousness, and seizure [1]. Patients who show neurological symptoms included cases with or without pre-existing neurological disorders [2]. While on intensive care units, patients showed agitation, confusion, and corticospinal tract signs such as enhanced tendon reflexes and clonus. In mild to moderate disease cases, patients reported olfactory $(85.6 \%)$ and gustatory $(88.0 \%)$ dysfunctions. Importantly, in about $11 \%$ of patients, anosmia occurred prior to any other clinical symptoms [3]. COVID-19 can further lead to changes of coagulation and, in particular, to inflammation-induced disseminated intravascular coagulation (DIC). Together with endothelial dysfunction, DIC can cause cerebrovascular ischemia even in young patients, many of whom suffer from large vessel ischemic stroke $[4,5]$. Overall, this may also be influenced by the severity of the COVID-19 infection, as $5.7 \%$ of the severe cases suffered from ischemic stroke [1] or had pre-existing vascular risk factors, especially in the elderly, in whom ischemic stroke rather occurred as 
a delayed complication [6, 7]. Additionally, as sub-acute signs that occurred 3-10 days after the development of Covid-19 symptoms, Guillain-Barré syndrome [8] and Miller-Fisher syndrome [9] cases have been reported. Also, clinically striking are cases of Kawaski-like multisystem inflammatory syndromes now being recognized in children and teenagers.

\section{Main text}

There are at least four possible pathogenic mechanisms that may account for the detrimental effect of COVID19 on the CNS: (1) direct viral encephalitis, (2) systemic inflammation, (3) peripheral organ dysfunction (liver, kidney, lung), and (4) cerebrovascular changes. In most cases, however, neurological manifestations of COVID19 may arise from a combination of the above.

Any one or a combination of these mechanisms put COVID-19 survivors at risk for developing long-term neurological consequences, either by aggravating a preexisting neurological disorder or by initiating a new disorder. This concern is supported by findings that show that one third of patients at the time of discharge have evidence of cognitive impairment and motor deficits [2]. This is particularly relevant because overall COVID-19 clinically affects the elderly most severely [10]. There is a large overlap of the age range when people typically develop neurodegenerative or cerebrovascular disease and the age of risk for the most several COVID-19 infections. This overlap argues that there is a compelling need for prospective neurological surveillance and care.

COVID-19 is associated with a severe innate immune response and sustained rise of systemic cytokine levels. Importantly, this innate immune response has been suggested to drive and predict mortality and severity [11]. Cytokines and related inflammatory mediators found to be elevated include interleukin-1 $\beta$, interleukin-2, interleukin2 receptor, interleukin-4, interleukin-10, interleukin-18, interferon- $\gamma$, C-reactive protein, granulocyte colonystimulating factor, interferon- $\gamma$, CXCL10, monocyte chemoattractant protein 1, macrophage inflammatory protein $1-\alpha$, and tumor necrosis factor- $\alpha[10,12]$. Concomitantly, most patients show signs of $\mathrm{T}$ cell exhaustion with lower lymphocyte counts. The fact that systemic inflammation has been shown to promote cognitive decline and neurodegenerative disease makes it likely that COVID-19 survivors will experience neurodegeneration in the following years $[13,14]$. Of note, cytokine levels can predict the subsequent occurrence of hippocampal atrophy in patients that experience severe sepsis [15]. In keeping with this, the most frequent clinical presentation of COVID-19 is the development of acute respiratory distress syndrome (ARDS) [16], the latter being, along with chronic ventilation, highly associated with subsequent cognitive decline, executive dysfunction, and reduced quality of life, often persisting months and years after hospital discharge [17] reviewed in [18]. The causative role of systemic inflammatory damage to the brain is further supported by the fact that none of the cerebrospinal fluid samples investigated in the study by Helms et al. found evidence for a direct presence of SARS-CoV-2 in the cerebrospinal fluid [2]. That being said, one should not ignore the potential importance of the single case report of direct viral infiltration of the brain and viral encephalitis, either by hematogenous or neuronal retrograde dissemination [19].

Evidence from murine lung injury models and ARDS patient samples emphasize the role of the NLRP3 inflammasome in the pathogenesis and detrimental outcome of ARDS [20, 21]. In keeping with this, the coronavirus ORF3a protein has been shown to induce NLRP3 inflammasome activity [22]. Moreover, ventilation-induced hypercapnia has been experimentally shown to lead to cognitive impairment in a NLRP3 inflammasomeinterleukin-1 $\beta$-dependent manner [23]. Given the above cytokine findings in COVID-19 patients and in particular the rise of interleukin-1 $\beta$ and interleukin-18, it seems highly likely that COVID-19 patients suffer from NLRP3 inflammasome activation. This activation and the subsequent increased activity of proinflammatory immune pathways are likely to exert a negative impact on cerebral homeostasis and function.

This conclusion is based on not only epidemiological evidence but also on experiments that showed that systemic, NLRP3 inflammasome-mediated inflammation adversely affects beneficial immune functions in the brain and thereby causes the pathological accumulation of neurodegeneration-associated peptides such as fibrillar amyloid- $\beta$ [24]. Thus, both peripheral and central induction of the NLRP3 inflammasome can directly induce or aggravate neurodegenerative processes that lead to functional impairment in $\mathrm{AD}$ [25] or strongly contribute to the spreading of pathology and thus the progression of the disease [26]. The recent finding showing that NLRP3-driven and interleukin-1 $\beta$-mediated modulation of phosphokinases and phosphatases largely accounts for the pathological formation of neurofibrillary tangles in murine models of tauopathy raises the concern that COVID-19 patients are likely to experience an induction or strong aggravation of neurodegenerative processes [27].

\section{Conclusion}

Evidence strongly suggests that patients surviving COVID-19 are at high risk for subsequent development of neurological disease and in particular Alzheimer's disease. Neurologists, psychiatrists, and caregivers should be alerted to a possible increase in such cases in COVID-19 survivors. Prospective studies are needed to 
investigate potential correlations between acute and subacute COVID-19 infections and long-term neurological sequalae in this patient cohort.

\section{Abbreviations}

AD: Alzheimer's disease; ARDS: Acute respiratory distress syndrome; COVID19: Coronavirus SARS-CoV-2; CSF: Cerebrospinal fluid; NLRP3: NOD-, LRR-, and pyrin domain-containing protein 3; ORF3a: Not characterized yet (UNIPROT)

\section{Acknowledgements}

This work was supported by the NIH grant (R01 AG059752-02) to DG and MTH.

\section{Authors' contributions}

All authors contributed equally to this viewpoint article. All authors read and approved the final manuscript.

\section{Funding}

Not applicable.

\section{Availability of data and materials}

Not applicable.

\section{Ethics approval and consent to participate}

Not applicable.

\section{Consent for publication}

Not applicable.

\section{Competing interests}

The authors declare that they have no competing interests.

\section{Author details}

${ }^{1}$ Department of Neurodegenerative Disease and Geriatric Psychiatry, University of Bonn, Bonn, Germany. ${ }^{2}$ German Center for Neurodegenerative Disease, Bonn, Germany. ${ }^{3}$ Division of Infectious Diseases and Immunology, University of Massachusetts Medical School, Worcester, MA 01605, USA. ${ }^{4}$ Institute for Innate Immunity, University of Bonn, Bonn, Germany. ${ }^{5}$ Translational Neuroscience, College of Human Medicine, Michigan State University, Grand Rapids, USA. ${ }^{6}$ Department of Neurology, University of Massachusetts Medical School, Worcester, MA 01605, USA.

Received: 6 May 2020 Accepted: 27 May 2020

Published online: 04 June 2020

\section{References}

1. Mao L, Jin H, Wang M, Hu Y, Chen S, He Q, et al. Neurologic manifestations of hospitalized patients with coronavirus disease 2019 in Wuhan, China. JAMA Neurol. 2020:e201127.

2. Helms J, Kremer S, Merdji H, Clere-Jehl R, Schenck M, Kummerlen C, et al. Neurologic features in severe SARS-CoV-2 infection. N Engl J Med. 2020: NEJMc2008597.

3. Lechien JR, Chiesa-Estomba CM, De Siati DR, Horoi M, Le Bon SD, Rodriquez $A$, et al. Olfactory and gustatory dysfunctions as a clinical presentation of mild-to-moderate forms of the coronavirus disease (COVID-19): a multicenter European study. Eur Arch Otorhinolaryngol. 2020;6:1-11.

4. Gonzalez-Pinto T, Luna-Rodriguez A, Moreno-Estebanez A, Agirre-Beitia G, Rodriguez-Antiguedad A, Ruiz-Lopez M. Emergency room neurology in times of COVID-19: malignant ischemic stroke and SARS-COV2 infection. Eur J Neurol. 2020:ene.14286.

5. Oxley TJ, Mocco J, Majidi S, Kellner CP, Shoirah H, Singh IP, et al. Largevessel stroke as a presenting feature of Covid-19 in the young. N Engl J Med. 2020;382(20):e60.

6. Beyrouti R, Adams ME, Benjamin L, Cohen H, Farmer SF, Goh YY, et al. Characteristics of ischaemic stroke associated with COVID-19. J Neurol Neurosurg Psychiatry. 2020:jnnp-2020-323586.

7. Viguier A, Delamarre L, Duplantier J, Olivot JM, Bonneville F. Acute ischemic stroke complicating common carotid artery thrombosis during a severe COVID-19 infection. J Neuroradiol. 2020:S0150-9861(20)30159-0.
8. Toscano G, Palmerini F, Ravaglia S, Ruiz L, Invernizzi P, Cuzzoni MG, et al. Guillain-Barre syndrome associated with SARS-CoV-2. N Engl J Med. 2020: NEJMc2009191.

9. Gutierrez-Ortiz C, Mendez A, Rodrigo-Rey S, San Pedro-Murillo E, BermejoGuerrero L, Gordo-Manas R, et al. Miller fisher syndrome and polyneuritis cranialis in COVID-19. Neurology. 2020. https://doi.org/10.1212/WNL. 0000000000009619

10. Chen G, Wu D, Guo W, Cao Y, Huang D, Wang H, et al. Clinical and immunological features of severe and moderate coronavirus disease 2019. J Clin Invest. 2020;130:2620-9.

11. Huang C, Wang Y, Li X, Ren L, Zhao J, Hu Y, et al. Clinical features of patients infected with 2019 novel coronavirus in Wuhan, China. Lancet. 2020:395(10223):497-506.

12. Mehta P, McAuley DF, Brown M, Sanchez E, Tattersall RS, Manson JJ, et al. COVID-19: consider cytokine storm syndromes and immunosuppression. Lancet. 2020;395(10229):1033-4.

13. Iwashyna TJ, Ely EW, Smith DM, Langa KM. Long-term cognitive impairment and functional disability among survivors of severe sepsis. JAMA. 2010; 304(16):1787-94.

14. Widmann CN, Heneka MT. Long-term cerebral consequences of sepsis. Lancet Neurol. 2014;13(6):630-6.

15. Lindlau A, Widmann CN, Putensen C, Jessen F, Semmler A, Heneka MT. Predictors of hippocampal atrophy in critically ill patients. Eur J Neurol. 2015;22(2):410-5.

16. Rodriguez-Morales AJ, Cardona-Ospina JA, Gutierrez-Ocampo E, VillamizarPena R, Holguin-Rivera Y, Escalera-Antezana JP, et al. Clinical, laboratory and imaging features of COVID-19: a systematic review and meta-analysis. Trave Med Infect Dis. 2020;34:101623.

17. Girard TD, Thompson JL, Pandharipande PP, Brummel NE, Jackson JC, Patel $M B$, et al. Clinical phenotypes of delirium during critical illness and severity of subsequent long-term cognitive impairment: a prospective cohort study. Lancet Respir Med. 2018;6(3):213-22.

18. Sasannejad C, Ely EW, Lahiri S. Long-term cognitive impairment after acute respiratory distress syndrome: a review of clinical impact and pathophysiological mechanisms. Crit Care. 2019;23(1):352.

19. Zhou L, Zhang M, Wang J, Gao J. Sars-Cov-2: underestimated damage to nervous system. Travel Med Infect Dis. 2020;101642.

20. Jones HD, Crother TR, Gonzalez-Villalobos RA, Jupelli M, Chen S, Dagvadorj J, et al. The NLRP3 inflammasome is required for the development of hypoxemia in LPS/mechanical ventilation acute lung injury. Am J Respir Cell Mol Biol. 2014;50(2):270-80.

21. Feng Z, Qi S, Zhang Y, Qi Z, Yan L, Zhou J, et al. Ly6G+ neutrophil-derived miR-223 inhibits the NLRP3 inflammasome in mitochondrial DAMP-induced acute lung injury. Cell Death Dis. 2017:8(11):e3170.

22. Siu KL, Yuen KS, Castano-Rodriguez C, Ye ZW, Yeung ML, Fung SY, et al. Severe acute respiratory syndrome coronavirus ORF3a protein activates the NLRP3 inflammasome by promoting TRAF3-dependent ubiquitination of ASC. FASEB J. 2019:33(8):8865-77.

23. Ding $H G$, Deng $Y Y$, Yang $R Q$, Wang $Q S$, Jiang $W Q$, Han $Y L$, et al. Hypercapnia induces IL-1 beta overproduction via activation of NLRP3 inflammasome: implication in cognitive impairment in hypoxemic adult rats. J Neuroinflammation. 2018;15(1):4.

24. Tejera D, Mercan D, Sanchez-Caro JM, Hanan M, Greenberg D, Soreq H, et al. Systemic inflammation impairs microglial A $\beta$ clearance through NLRP3 inflammasome. EMBO J. 2019:38(17):e101064.

25. Heneka MT, Kummer MP, Stutz A, Delekate A, Schwartz S, Vieira-Saecker A, et al. NLRP3 is activated in Alzheimer's disease and contributes to pathology in APP/PS1 mice. Nature. 2013;493(7434):674-8.

26. Venegas C, Kumar S, Franklin BS, Dierkes T, Brinkschulte $R$, Tejera D, et al. Microglia-derived ASC specks cross-seed amyloid-beta in Alzheimer's disease. Nature. 2017:552(7685):355-61.

27. Ising C, Venegas C, Zhang S, Scheiblich H, Schmidt SV, Vieira-Saecker A, et al. NLRP3 inflammasome activation drives tau pathology. Nature. 2019; 575(7784):669-73

\section{Publisher's Note}

Springer Nature remains neutral with regard to jurisdictional claims in published maps and institutional affiliations. 